\title{
L'alternance (néo)codique comme ressource communicative dans les formules de salutations: étude des échanges synchrones entre cyberscripteurs algériens
}

\section{Neo-Code switching as a communicative resource in salutations formulas: study of synchronous exchanges between Algerian cyber-scripters}

\author{
Hasna Sebiane ${ }^{1}$ a \\ ${ }^{1}$ Université Abou-Bakr Belkaid Tlemcen / Laboratoire DYLANDIMED (UABB) \\ 22, Rue Abi Ayad Abdelkrim, Fg Pasteur BP 119 Tlemcen, Algérie
}

\begin{abstract}
Résumé. Nous nous proposons de contribuer à la réflexion autour de la notion de ressources numériques en nous intéressant à la communication médiée par ordinateur. Nous nous intéresserons particulièrement au fonctionnement de l'alternance (néo)codique comme ressource langagière numérique qui participe lors de l'interaction à distance à l'organisation séquentielle des échanges entre cyberscripteurs algériens. En effet, les ressources langagières et linguistiques constituées à partir de nouveaux corpus de communication numérique écrite sont susceptibles d'intéresser les chercheurs en sciences du langage. Ce travail sera, de fait, consacré à l'analyse de l'alternance codique et de son aspect fonctionnel à partir des échanges synchrones écrits sur Facebook, où le choix des langues s'avère être accompli collaborativement par les cyberscripteurs. Cet accomplissement relève du processus d'alignement et d'ajustement codique, voire parfois de négociations mutuelles qui participent à la co-construction de la communication en ligne.
\end{abstract}

\begin{abstract}
We aim to contribute to the debate concerning the concept of digital resources. We intend to do this by focusing on computer-mediated communication. Since both language and linguistic resources that are built from several written digital communication corpora are likely to attract the attention of researchers in the field of linguistics, we are particularly interested in the functioning of code switching as a digital language resource that plays a part in sequentially organized distant interaction among Algerian cyber-scripters. This work will be devoted to the analysis of code switching and its functional aspects in written synchronous exchanges on Facebook, in which language choice is known to be collaboratively fulfilled by cyberscripters. This achievement stems from a code adjustment and alignment process, sometimes even from the mutual negotiations that are part of a coconstruction of online communication.
\end{abstract}

\footnotetext{
${ }^{a}$ Adresse de correspondance : sebiane.h02@gmail.com
} 


\section{Introduction}

La multiplicité des supports numériques, dans un monde dit de l'information et de la communication, représente " un centre de ressources planétaires dont la seule frontière est constituée par la langue d'expression » (Pothier, 2004 : 19) et un «eldorado » (Pierozak, 2010) de ressources pour les chercheurs qui s'intéressent aux interactions multimodales et plurilingues. Ainsi, la diversification des objets d'études amènent les chercheurs en sciences du langage à interroger les faits émergeants ainsi que les faits et les effets résultant de ce type de communication. Il s'agit, en fait, de ressources numériques en ligne qui, selon leurs configurations et leurs originalités, invitent les chercheurs à repenser la notion de ressource selon des angles de vues différents.

A priori, le paysage des communications numériques se présente comme un (cyber)espace propice à l'alternance des langues en présence, où chacune d'elles remplit des fonctions spécifiques. De même, les cyberscripteurs ${ }^{1}$ algériens disposent d'un répertoire "scripto-verbal plurilingue » (Ali-Bencherif, 2016: 33) qu'ils manipulent en fonction d'autres éléments de la situation de communication (Hymes, 1972). Néanmoins, nous n'allons pas envisager tous les aspects fonctionnels de l'alternance codique, nous nous limiterons aux formules de salutations (à savoir, les formules d'ouverture et de clôture) comme ressources langagières entièrement ritualisées relevant, de facto, de la « cyberpolitesse », telles qu'elles ont été abordées par Kerbrat-Orecchioni (2007).

Cette étude sera donc consacrée à l'analyse des formules de salutations - à partir des échanges synchrones sur Facebook entre cyberscripteurs algériens - qui constituent, à notre sens, un aspect important dans la communication médiée par ordinateur. Nous voulons rendre compte des ressources langagières mobilisées à travers cette activité interactionnelle marquée de part et d'autres par l'usage fréquent de l'alternance (néo)codique ${ }^{2}$, en termes d'influences subies et d'influences exercées pour la production du sens dans la communication en ligne.

À travers une analyse des ressources d'un corpus composé d'échanges, nous traiterons les questions suivantes: comment des ressources langagières numériques sont-elles coconstruites dans les échanges synchrones entre les cyberscripteurs algériens ? Dans quelle mesure l'alternance codique devient-elle une ressource pour l'organisation séquentielle des échanges en ligne? Comment se fait le choix des ressources langagières dans les formules de salutations?

Ce travail s'inscrit dans le champ de l'analyse des interactions en ligne, et se focalise sur la question de la mobilisation des ressources langagières numériques provenant de différents systèmes linguistiques dans une communication médiée par ordinateur.

\footnotetext{
${ }^{1}$ Dans les travaux sur la communication médiée par ordinateur, on retrouve assez souvent l'emploi du terme « internaute». Le terme de «cyberscripteur» renvoie, de fait, à un locuteur-scripteur qui se trouve dans un cyberespace (Internet) derrière un écran pour échanger avec son interlocuteur en se servant du clavier et des moyens qu'offre la machine pour communiquer.

${ }^{2}$ Nous entendons par l'alternance néo-codique les nouvelles pratiques d'écriture via écran, qui tirent leur marque de la langue parlée, repérée ainsi au niveau de l'orthographe. Autrement dit, c'est une nouvelle forme de langue métissée produite à partir d'un contact entre la technologie et la langue parlée (Develotte et Gee, 2005 : 153). Par ailleurs, nous estimons que ces néo-codages sont le résultat d'une co-construction des cyberscripteurs.
} 


\section{Corpus et démarche méthodologique}

Nous allons présenter dans ce qui suit, les orientations méthodologiques qui nous ont conduite à définir notre démarche et à constituer notre corpus d'étude.

\subsection{La communication médiée par ordinateur: enjeux méthodologiques pour l'analyse des ressources numériques}

L'analyse de la communication médiée par ordinateur a un avantage méthodologique qui réside dans la facilité de la constitution d'un corpus représentatif et significatif, enregistrable et «transcrit» préalablement par les participants (Marcoccia, 2016:38). En effet, ces corpus naturels et déjà transcrits sont considérés comme des ressources numériques (souvent sous forme textuelle) correspondant intégralement à «l'expérience réelle des participants» (Wascul \& Douglass, 1996 ; cité par Marcoccia, 2016 : 38). A fortiori, le chercheur n'est pas confronté au «paradoxe de l'observateur » au sens de Labov (1976); au contraire il peut être pratiquement certain d'avoir recueilli un corpus authentique, non influencé et donc significatif de la pratique spontanée des interlocuteurs. De cette manière, on peut ainsi avoir « accès à la façon dont les gens se servent du langage quand on ne les observe pas » (Labov, 1976 ; cité par Pierozak 2010 : 25).

Cependant, nous relevons une sorte de paradoxe lié à l'accès au contexte de production des messages échangés. En effet, la nature «déspacialisée » et donc «déterritorialisée » de la communication en ligne (Marcoccia, 2011: 96) risque de bloquer les chercheurs qui travaillent sur des corpus constitués à partir de ressources numériques. Or, l'intérêt même d'analyser ce type de communication va de pair avec un ancrage de la production des messages dans un contexte social bien défini (Marcoccia, 2016).

La communication médiée par ordinateur est souvent évoquée en termes de «déspacialisation» et a priori, analysée comme une communication "spatialement décontextualisée » en raison de l'absence physique des participants. Ainsi, traitant les espaces de la conversation visiophonique en ligne, Marcoccia souligne que les participants produisent des demandes de localisation, en utilisant la formule rituelle «t'es où ? » et de cette manière, "les contextes spatiaux dans lesquels se situent les [participants] ont des effets sur la communication en ligne» (Marcoccia, 2011: 114). À cet effet, nous considérons la communication médiée par ordinateur comme un espace de socialisation langagière où les échanges synchrones entre cyberscripteurs algériens sont bien contextualisés. Néanmoins, les éléments du contexte pris en compte sont ceux qui sont accessibles à travers les ressources langagières numériques.

Toujours est-il que l'alternance des tours d'écriture n'est pas toujours évidente à appréhender. Cette alternance est le résultat des procédés d'affichage du système généré par la machine (en l'occurrence, par le système du réseau Facebook) mais en réalité, cette alternance des messages échangés peut être différente par rapport au processus d'affichage, c'est-à-dire que les messages peuvent être éloignés au moment de leur production. 


\subsection{Méthodologie}

Notre approche sociolinguistique des interactions verbales et de la communication médiée par ordinateur, où l'alternance codique sera abordée en tant que ressource langagière numérique provenant de différents systèmes linguistiques et traitée par les cyberscripteurs dans une relation de contiguïté, nous a conduit à adopter une démarche logocentrée du traitement du corpus des conversations de Facebook.

Toutefois, la constitution d'un corpus textuel, focalisé sur la dimension linéaire et opérant par extraction des matériaux linguistiques isolés de leurs contextes de production, a pour conséquence d'extraire les données de leur environnement numérique. Ce genre de corpus - qualifié de «verbo-centriste» (Marcoccia, 2016:59) et construit à partir d'un dispositif de la communication numérique - permet l'archivage des ressources dans un fichier de texte standard. Ce traitement logocentré rend le corpus plus facilement manipulable et permet une analyse centrée uniquement sur les pratiques langagières écrites des cyberscripteurs.

\subsection{Description du corpus}

Il faut préciser que notre étude suit une démarche exploratoire de l'alternance (néo)codique à visée compréhensive et se base sur un corpus composé de 1066 tours d'écriture ${ }^{4}$ bi ou plurilingue, qui a été établi par un tri manuel à partir d'une base de données conversationnelles d'un support numérique : la discussion instantanée du réseau social Facebook. Le recueil s'est fait par le biais d'une requête/sollicitation auprès de sujets âgés entre 20 et 30 ans sans présélection du genre des auteurs des conversations ${ }^{5}$. Effectivement, nous avons cherché à obtenir un consentement des participants (des cyberscripteurs) dont les messages seront analysés.

Par ailleurs, aussi étendu que soit un corpus de la communication médiée par ordinateur, il ne peut être objet d'étude que s'il est limité. A fortiori, notre corpuséchantillon, sélectif et significatif pour l'étude de l'alternance (néo)codique dans les échanges synchrones, nous a conduit à écarter les ressources non-linguistiques (émoticônes, smileys, images, etc.) pour nous focaliser uniquement sur le matériau langagier des cyberscripteurs $^{6}$. En effet, la communication médiée par ordinateur est d'autant plus soumise à une variation orthographique et "permet de comprendre que ce [type de productions écrites] varie des formes les plus standardisées aux formes les plus déviantes »

\footnotetext{
${ }^{4}$ La communication synchrone se rapproche de la communication face à face, dans la mesure où elle se fonde sur l'alternance des rôles d'émetteur et de récepteur. Ceci dit, la notion de tour de parole, propre à la conversation où deux interlocuteurs interagissent pour construire ensemble une interaction, peut être appliquée à notre corpus. En effet, la messagerie instantanée, de nature dialogale, se réalise à partir de l'alternance des «tours d'écriture », décalés dans le temps et dans l'espace.

${ }^{5}$ Dans le cas de cette étude, nous n'avons pas pris en compte la variable sociale sexe, car nous avons jugé cette variable non-pertinente pour l'analyse des pratiques langagières des cyberscripteurs étudiés.

${ }^{6} \mathrm{Il}$ faut noter que ce type de traitement du corpus peut s'avérer limité à quelques cas de figures pour l'étude des salutations à travers une communication médiée par ordinateur. En effet, nous ne prenons pas en compte le matériau sémiotique pour l'étude des formes de salutations, mais nous nous centrons sur les productions écrites des cyberscripteurs, ce qui du même coup, nous facilite la manipulation de traitement du corpus.
} 
(Liénard, 2014 : 147). Les TIC ont permis aux cyberscripteurs qui n'auraient jamais tant écrit, d'écrire tout simplement dans leurs langues maternelles ${ }^{7}$ (Liénard, 2014).

Notre corpus échantillonné présente un recueil de conversations bi-plurilingues, comprenant un nombre suffisant de messages, voire de tours d'écriture, et jugé donc significatif pour une analyse exploratoire. Les données numériques collectées ont été anonymisées en utilisant les initiales à la place des noms des auteurs des conversations.

\section{Redéfinition de la notion de ressources}

Les méthodes de création de ressources langagières via les dispositifs numériques sont aujourd'hui très répandues. La notion de ressources a connu une extension avec l'apparition des TIC, ce qui nécessite de clarifier ses différents contextes d'emploi. Il s'agit, en effet, d'une notion trop souvent employée dans les différents domaines qui traitent des questions didactiques et sociolinguistiques (ce qui est le cas de cette étude). Bien qu'elle soit rarement définie, nous cherchons à l'appréhender à partir d'un dispositif de communication numérique écrite (la discussion instantanée de Facebook) afin de rendre compte des nouvelles pratiques (socio)langagières. Quelle est alors la nature de ces changements survenus avec le développement des TIC du point de vue des ressources?

Il faut d'emblée souligner qu'en essayant de cerner la notion de ressources dans le domaine de l'usage des TIC, le problème qui se pose est celui de l'excès des ressources en ligne, voire de ressources numériques, et de trouver les éléments les plus pertinents que nous cherchons.

La notion de ressources, entendues comme des données de toutes sortes (écrites, audio, visuelles), quelle que soit leur forme (numérique ou autre), nous en distinguons deux définitions pertinentes, en didactique des langues et en linguistique. Dans sa définition des « ressources d'apprentissage», Holec les considère comme un "ensemble des moyens (documents et tâches) utilisés par un apprenant de langue pour réaliser les acquisitions définies par les objectifs qu'il s'est fixé » (1995, cité par Pothier, 2004 : 18), d'une part. D'autre part, les ressources linguistiques dans le domaine des TIC sont « tous les types de données relatives à la langue, accessibles dans un format électronique, et utilisées pour le développement des systèmes de traitement de la parole et du texte dans des applications en technologies de l'information $»^{8}$.

En interrogeant la notion de ressources à travers notre corpus, nous pouvons appréhender ce concept de deux façons différentes; d'abord, il s'agit des nouvelles formes de la communication en ligne (il est question, ici, de la discussion instantanée de Facebook) qui s'appuient sur des solutions techniques qu'offre l'outil informatique pour permettre l'enregistrement d'un nombre considérable de données numérisées, par le biais de l'archivage systématique des messages échangés entre les cyberscripteurs. Ensuite, il s'agit de phénomènes socio-langagiers originaux qui ont émergés de l'utilisation massive des nouveaux modes de la communication informatisée. En outre, les ressources numériques émanent des interactions elles-mêmes dont les productions des messages deviennent à leur tour des ressources potentiellement exploitables.

\footnotetext{
${ }^{7}$ L'arabe algérien, qui est une langue décrite grâce au développement des TIC, n'est ni enseignée, ni codifiée orthographiquement. Ceci dit, les cyberscripteurs algériens produisent les mots dans leur langue maternelle comme ils les prononcent.

${ }^{8}$ www.technolangue.net
} 
Nous pouvons ainsi représenter la notion de ressources et son rapport aux TIC à partir du modèle suivant :

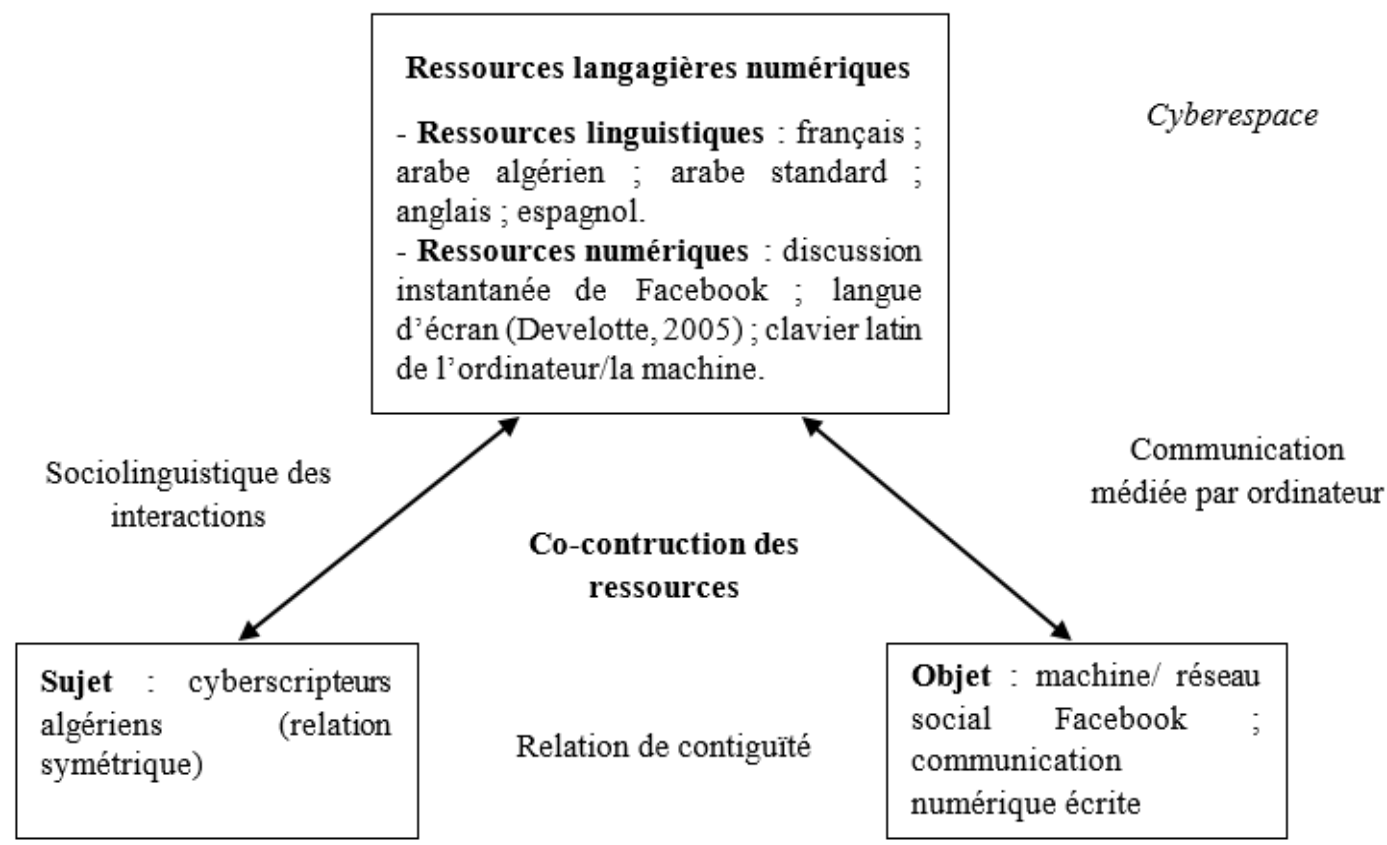

Figure 1. Ressources dans un modèle de dispositif de la communication médiée par ordinateur

Ce modèle schématise les éléments d'une conception systémique du dispositif de la communication médiée par ordinateur et des ressources linguistiques et langagières mobilisées à travers la constitution et le traitement d'un corpus numérique, qui sont une combinatoire de ressources articulées dans une dynamique en fonction des comportements langagiers et des répertoires scripto-verbaux des cyberscripteurs, traitée dans une relation de contiguïté.

\section{Analyses et discussion des données}

\subsection{Choix et gestion des langues dans la discussion sur Facebook, un indice de compétence bi-plurilingue ?}

Le contexte sociolinguistique algérien est caractérisé par la coexistence de plusieurs langues et/ou variétés de langues en présence : l'arabe algérien comme langue maternelle des locuteurs et langue de communication au quotidien; le berbère ${ }^{9}$ représente également la langue maternelle des berbérophones et est employée par une communauté linguistique minoritaire ; l'arabe standard, langue officielle du pays, demeure une langue réservée à la religion, à l'administration et aux institutions formelles ; enfin les langues étrangères,

\footnotetext{
9 La langue berbère est totalement absente dans notre corpus, nous n'avons rencontré aucune
} transcription orthographique de cette langue. 
comprenant principalement le français, qui conserve une place importante au sein de la société algérienne, l'anglais et l'espagnol, dont l'usage reste encore faible.

Si la situation sociolinguistique en Algérie est marquée par la coexistence et le contact de plusieurs langues, il en est de même pour les situations de communication numérique. Etant bi- ou plurilingues, les cyberscripteurs algériens utilisent au moins deux langues (l'arabe algérien et le français) - dont certaines ne sont pas codifiées orthographiquement ${ }^{10}$ - dans leurs échanges en ligne. Nous soulignons, à ce propos, que les TIC jouent le rôle de médiateurs linguistiques (Chardenet, 2005 : 237) et stimulent de part et d'autre l'usage des langues étrangères, et davantage celui des langues maternelles et/ou minoritaires ${ }^{11}$.

Dans notre corpus, les langues sont gérées et investies différemment selon les compétences linguistiques caractérisant chaque cyberscripteurs. De même, le parler biplurilingue (un phénomène qui résulte des échanges de la communication en ligne) représente un mode commun et permet le maintien de la communication. Ainsi, l'alternance des codes diminuent les asymétries entre les sujets n'ayant pas les mêmes compétences linguistiques, voire le même répertoire scripto-verbal ${ }^{12}$.

Le français se trouve principalement alterné avec l'arabe algérien et moins fréquemment avec l'arabe standard. Il faut également rappeler que l'alternance du français avec l'arabe algérien est une pratique courante dans les interactions verbales des locuteurs algériens. Par ailleurs, la première caractéristique notable du corpus qui émane de nos observations générales est la présence dominante du français et de la graphie latine à côté de l'arabe algérien. Cette variété de la langue arabe - utilisée uniquement à l'oral dans les usages ordinaires de la communication face à face - est transcrite/orthographiée par le biais de la graphie latine. Il s'agit, en fait, d'un contact de langue parlée/langue écrite, et d'un indice d'une compétence bi-plurilingue en construction.

\subsection{Le fonctionnement socio-numérique et interactif de l'alternance codique}

Comme mentionné supra, nous nous limitons dans cette étude aux formules de salutations (les séquences d'ouverture et de clôture). L'ouverture constitue un moment crucial pour l'organisation séquentielle de l'interaction (Mondada, 2007 : 184), où le choix des langues est accompli en co-collaboration par les cyberscripteurs. Les alternances (néo)codiques comme ressources analysées à partir des échanges synchrones, sont la plupart du temps retrouvées dans les formules d'ouverture et de clôture, et particulièrement sous forme d'expressions figées ou de mots isolés.

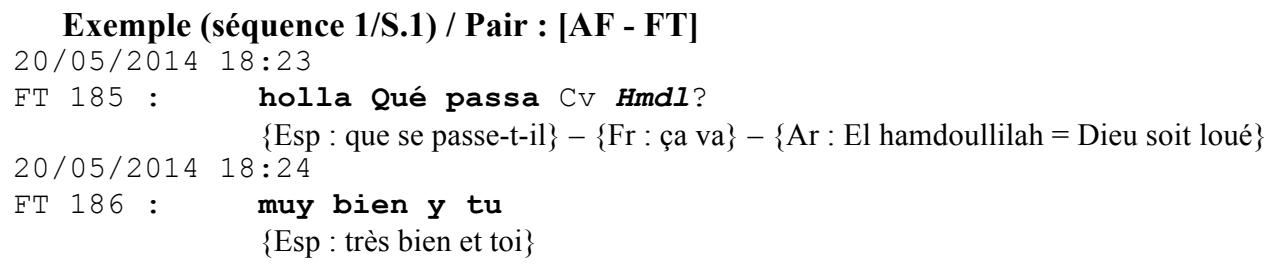

\footnotetext{
${ }^{10}$ Malgré les contraintes technologiques, les cyberscripteurs algériens se sont appropriés les mécanismes de la communication médiée par ordinateur en employant, à l'aide du clavier « latin », leur langue maternelle (l'arabe algérien). Le choix de cette langue découle de la nature conviviale et amicale de la communication en ligne (Atifi, $2007: 40$ ).

${ }^{11}$ À ce propos, Calvet (2002) remarque que l'anglais, langue dominante sur Internet, subit une baisse lente de son utilisation pour laisser place aux langues supranationales, ainsi qu'à d'autres langues plus ou moins minoritaires. Seulement, le problème qui se pose est lié au système orthographique des langues et leur respect fluctuants sur Internet.

${ }^{12}$ Le terme de répertoire scripto-verbal, pris dans le sens du répertoire verbal de Gumperz (1982), est employé ici pour désigner une écriture pouvant s'apparenter à celle de l'oral.
} 
L'exemple ci-dessus (S.1) illustre un cas d'exception de l'utilisation de l'alternance codique figurant dans le corpus, une adaptation de formes empruntés à une autre langue (l'espagnol : Esp), dont la structure morphosyntaxique est plus ou moins proche de celle du français, d'une part ; d'autre part, l'utilisation d'un élément isolé, d'une expression figée, comme (S.1) « $h m d l »$ de l'arabe standard, pour invoquer en quelque sorte Dieu, est transcrit dans une graphie latine, sous forme de squelette consonantique. À cet égard, dans son approche du code switching, Auer (1995) attribue aux participants des compétences et des préférences linguistiques auxquelles ceux-ci s'ajustent et leurs interlocuteurs s'alignent avec ce choix en intervenant dans la discussion avec une prise de tour d'écriture en espagnol comme l'exemple (S.1) cité ci-dessus « muy bien y tu».

A priori, l'ouverture est le lieu où le choix des langues s'accomplit par les cyberscripteurs au sein des échanges en ligne, leur permettant ainsi de s'aligner mutuellement. L'alternance codique dans les séquences d'ouverture et de clôture ${ }^{13}$ concerne surtout des expressions courtes (en arabe algérien ou en français), elle consiste en un seul mot et est produite aussi souvent que possible dans une «langue d'écran ${ }^{14}$ (Develotte et Gee, 2005).

Pour des raisons d'espace, nous ne présentons ci-dessous que quelques exemples des séquences d'ouverture et de clôture de notre corpus qui illustrent la régulation et la ritualisation de choix de ressources linguistiques et langagières :

Exemple S.2 /pair : [NE - IS]

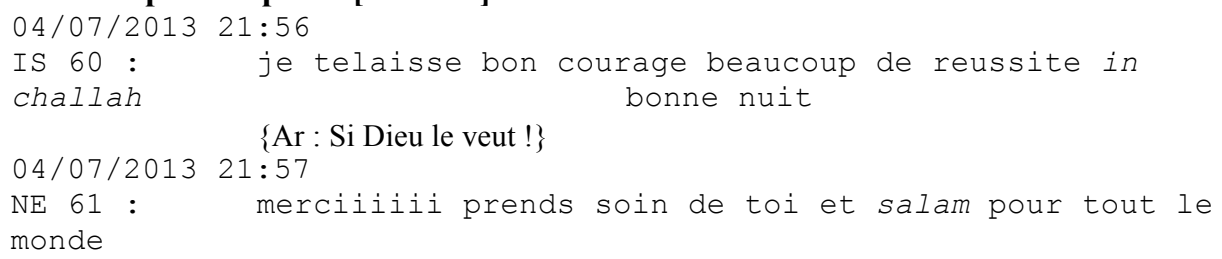

Exemple S.3 /pair : [NE - YRmc]

$\{$ Ar : bonjour $\}$

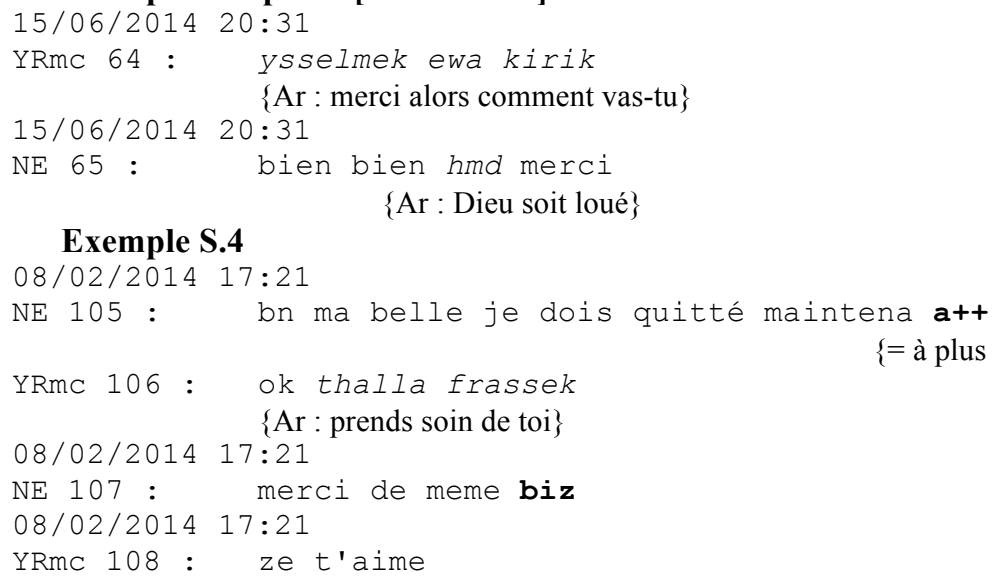

\footnotetext{
${ }^{13}$ Il faut noter que les salutations dans les séquences d'ouvertures sont plus fréquentes dans notre corpus. Certaines conversations s'achèvent sans séquence de clôture.

${ }^{14}$ Develotte et Gee (2005) font référence à la langue d'écran par opposition aux langues naturelles, il en ressort des alternances néo-codiques caractérisées par des usages langagiers, situées entre l'oral et l'écrit et influencées entre autres par le caractère synchrone de la communication médiée par ordinateur.
} 


\section{Exemple S.5}

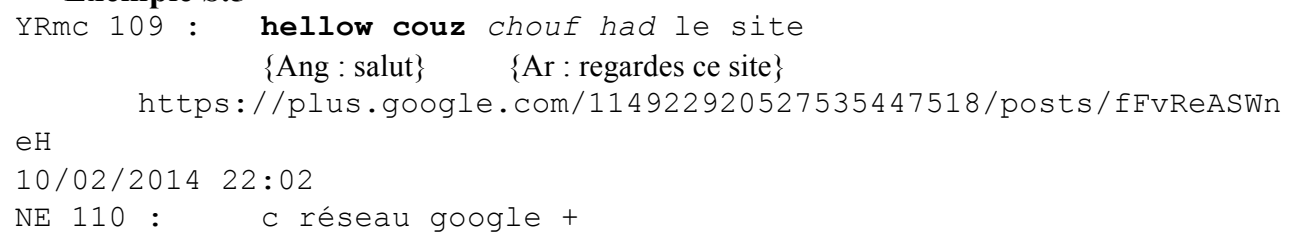

\section{Exemple S.6}

10/02/2014 22:08

YRmc 117 : oui deja nekhedmo biha

$\{\mathrm{Ar}$ : on travaille avec $\}$

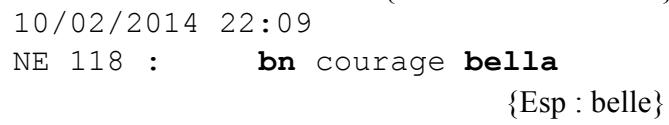

\section{Exemple S.7/ Pair : [AF - FT]}

04/05/2014 18:06

FT 205 : $\quad$ zinoo000000000000000000

04/05/2014 18:06

AF 206 : SIm toiiii Cv

$\{$ Ar : bonjour

04/05/2014 18:07

FT 207 : oui très très très bien

04/05/2014 18:07

AF 208 : Ouuuaaaaiiiiiiiiiil hmdl

$\{$ Ar : Dieu soit loué\}

$04 / 05 / 2014 \quad 18: 10$

FT 209 : et toi cv

04/05/2014 18:10

AF 210 : Hmdl trankil Au calme

$\{$ Ar : Dieu soit loué\}

$04 / 05 / 201418: 12$

FT 211 : $\quad$ c bien nchalah toujours comme ça $\{$ Ar: Si Dieu le veut\}

$04 / 05 / 2014 \quad 18: 12$

AF 212 : Tjr inchaaaaaaaAllah

\section{Exemple S.8}

$22 / 05 / 2014 \quad 18: 23$

AF 214 : Salam aleykoum ptite sœur ???? T là?

$\{$ Ar : Bonjour

$22 / 05 / 2014 \quad 18: 31$

\section{$\{$ Ar: Si Dieu le veut\}}

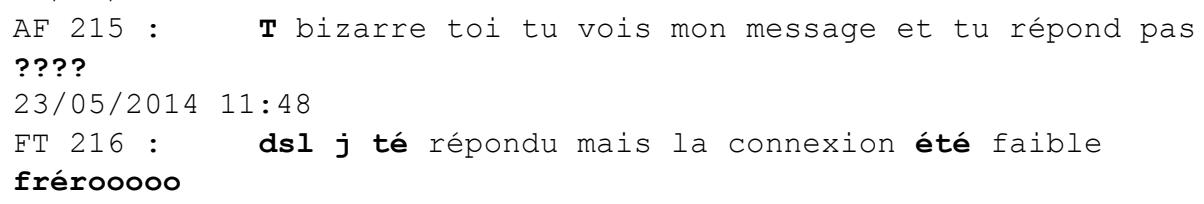




\section{Exemple S.9}

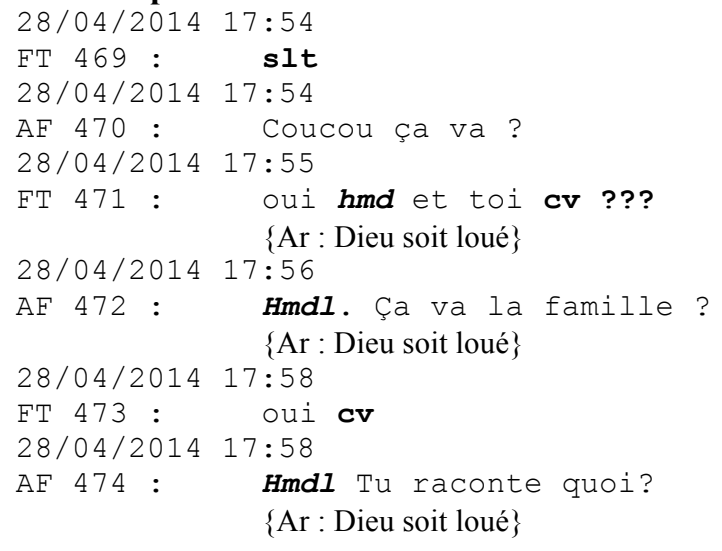

\section{Exemple S.10 /Pair : [RB - AB]}

30/09/2012 00:17

RB 572 : À la prochaine bye Asma !

30/09/2012 00:17

AB 573 : ok bay rachid bon8 thala frouhak

\section{Exemple S.11}

$\{\mathrm{Fr}$ : bonne nuit (langue d'écran)\} \{Ar: prends soin de toi\}

22/07/2014 14:31

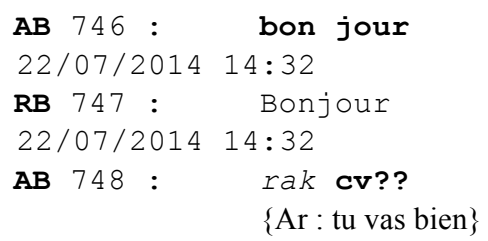

\section{Exemple S.12 / Pair : [YCC - SS]}

14/10/2014 23:45

SS 970 : yasmin bon 8 layla sa3ida nchalah

$14 / 10 / 2014 \quad 23: 46$

$\{\mathrm{Fr}$ : bonne nuit (langue d'écran)\} $\{\mathrm{Ar}$ : bonne nuit si Dieu le veut\}

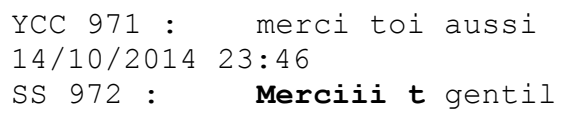

\section{Exemple S.13}

15/10/2014 11:14

SS 973 : Sbh nor

15/10/2014 11:15

\{Ar: bonjour\}

YCC 974 : hello

\{Ang: salut\} 


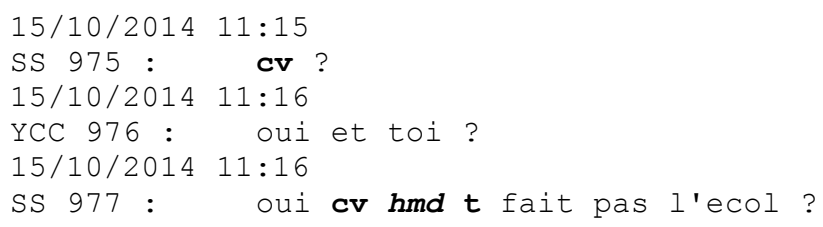

Les séquences d'ouverture et de clôture illustrent une certaine régulation, voire un ajustement codique quant au choix des ressources qui se fait principalement en arabe algérien et en français. Ce choix est relatif à la position des cyberscripteurs et à l'enchaînement séquentiel de l'activité interactionnelle en cours.

Parmi les exemples cités (S.1, S.5, S.9, S.13), les occurrences des expressions ou mots isolés de type «salut, coucou, hello, holla » combinés avec des éléments de l'arabe algérien «slm, hmdl» sont fréquents et leur prédominance sur les équivalents en arabe algérien représente un trait distinctif de la communication médiée par ordinateur. Ceci suggère que la prédominance du français est due au clavier latinisé de l'ordinateur/de la machine, mais aussi à la langue « officielle » et/ou de l'interface du réseau Facebook. Il n'en est pas moins que les raisons qui poussent les cyberscripteurs algériens à utiliser ces expressions isolées en français et dans d'autres langues étrangères relèvent d'une question d'économie ${ }^{15}$ (Pekarek Doehler, 2011) et ont un effet favorable à l'emploi de ces langues étrangères.

Un autre élément notable au sujet des ressources étudiées est que l'alternance codique, dans les formules de salutations, a également pour effet d'augmenter l'expressivité des messages. Les récurrences de salutation, des vœux, des expressions d'invocation à Dieu (S.2, S.3, S.7, S.9, S.11, S.12, S.13) dans les séquences d'ouverture et de clôture des échanges en ligne sont des énoncés souvent figés dans les pratiques langagières numériques des cyberscripteurs algériens (Ali-Bencherif, 2009 : 84) et permettent de distinguer la relation interpersonnelle ${ }^{16}$ entretenue entre ces derniers.

Dans les formules de salutations, l'alternance codique se caractérise par des usages à forte dominance phatique, mobilisés particulièrement par des formules de salutation, de vœux et d'expression culturelle (d'invocation à Dieu, comme par exemple «hmdl, inchallah »). Elle consiste le plus souvent en des expressions figées ou en un seul mot isolé. De cette manière, le caractère phatique de l'alternance codique offre la possibilité d'indexer son appartenance à une communauté ou à une culture spécifique. Désormais, l'alternance comme ressource langagière dans la communication numérique écrite est aussi un lieu de manifestation identitaire située au sein de l'activité interactionnelle à distance. Les formules de salutations relevant de l'arabe algérien font partie d'une ritualisation sociale communicative de la communication en ligne, tout comme les usages ordinaires.

En outre, l'emploi de l'alternance (néo)codique dispose ici d'un démarquage à valeur phatique, par rapport aux éléments du reste du message et sont fréquemment accompagnés de ressources typiques de la communication en ligne, comme les étirements graphiques «Merciiiiii, zinoooooooooooooooooooo, Ouuuuaaaaiiiiiiiiiiii, inchaaaaaaaaAllah », les

\footnotetext{
${ }^{15}$ Le recours à l'alternance codique serait motivé pour des raisons d'économie, de façon à ce que les cyberscripteurs bi-plurilingues puissent raccourcir la longueur de leur message par le choix de lexèmes les plus courts dans une langue (le français) ou une autre (l'arabe algérien) (Bautista, 2004 ; cité par Pekarek Doehler, 2011).

${ }^{16}$ La discussion instantanée de Facebook se particularise par la nature privée des échanges entre les cyberscripteurs et, par conséquent, de la relation interpersonnelle de ces derniers. Nous avons relevé des indices tels que «petite sœur», «cousin», «tata», etc., qui caractérisent la relation interpersonnelle entretenue entre eux.
} 
majuscules, les points d'exclamations ${ }^{17}$, les squelettes consonantiques « hmd, hmdl, slm, sbh, bn, cv, slt, cc, dsl, tjr », les logogrammes «b1, a++, bn8 » (qu'on retrouve dans la quasi-totalité des séquences analysées), ayant pour effet d'augmenter l'expressivité des échanges synchrones sur Facebook.

\subsection{Discussion: l'alternance codique comme ressource langagière numérique "routinisée "}

La communication numérique écrite mobilise de nouvelles pratiques d'utilisation de/s langue/s. Les cyberscripteurs disposent d'un répertoire scripto-verbal bi-plurilingue, leur permettant de développer et de renouveler des ressources qui sont à leur disposition (ressources linguistiques, langagières et numériques) pour atteindre des buts communicatifs communs. Dans les messages analysés et spécifiquement dans les séquences d'ouverture et de clôture, on remarque au minimum deux langues utilisées de manière alternées et parfois sous forme d'insertion, qui sont à leur tour alternées avec des procédés graphiques, de façon à constituer une langue d'écran au sens de Develotte et Gee (2005).

Le choix de langue/s est traité et accompli - dans ce type de contexte de la communication numérique écrite - au cours de l'organisation séquentielle des échanges en ligne (Mondada, 2007 :188). Dans ce sens, le choix des ressources effectué est parfois catégorisé par les cyberscripteurs comme une préférence linguistique, auxquelles des ajustements et des alignements codiques sont attribués à l'initiation d'une nouvelle séquence ou d'une activité interactionnelle.

De manière intéressante, la prédominance du français et d'autres langues étrangères (l'anglais et l'espagnol, par exemple) constituent un trait distinctif des séquences d'ouverture et de clôture de la communication en ligne, du fait du clavier latin de la machine et de la langue de l'interface (le français) du réseau social Facebook. Nous suggérons ainsi que les expressions isolées de type «slt», « hello », « holla » par exemple, font parties des routines communicatives (Pekarek Doehler, 2011: 11) spécifiques aux conversations instantanées de Facebook, relevant de part et d'autre d'un répertoire propre à la communication en ligne et puisant par-là, dans l'utilisation du français sur les équivalents en arabe algérien. Dans ce cadre, l'alternance (néo)codique est considérée comme une ressource langagière numérique, reconnue comme telle par les cyberscripteurs algériens, traitée par ces derniers dans une relation de contiguïté comme un indice de contextualisation (Gumperz, 1982), celui du cyberespace (par référence à Facebook).

À cet effet, les salutations - configurées pour ce type d'échange spécifique aux TIC deviennent une séquence qui fonctionne comme une ressource numérique pour vérifier et assurer l'interconnectivité des interactants (nous illustrons par l'exemple S.8 cité supra) «Salam aleykoum (bonjour) ptite sœur???? T là ?». De la même manière, les cyberscripteurs reviennent fréquemment à leur langue maternelle (l'arabe algérien), qui marque une manifestation identitaire et une appartenance socioculturelle (notamment les invocations à Dieu). Les langues alternées dans les formules de salutations en arabe algérien et en français, sont considérées comme des séquences à fonction phatique et des « rituels d'accès mutuels » (Goffman, 1973).

\footnotetext{
${ }^{17}$ Les majuscules et les points d'exclamations ne figurent pas dans les exemples illustrés supra et sont peu fréquents dans les formules de salutations.
} 


\section{Conclusion}

L'approche sociolinguistique interactionnelle a permis de saisir dans ce mode de communication «déspacialisée », la réalisation des activités interactionnelles et langagières, en mettant l'accent sur la notion de ressources. Dans le cadre de cette étude, nous avons tenté d'analyser les alternances codiques qui caractérisent les pratiques langagières des cyberscripteurs algériens - à travers des échanges de la discussion instantanée de Facebook - dans les séquences d'ouverture et de clôture constituant des ressources langagières numériques actualisées. Celles-ci sont l'indice d'une compétence biplurilingue co-construite contribuant, a posteriori à l'élaboration d'un cadre d'échanges commun de la communication en ligne. En d'autres termes, les ressources langagières numériques peuvent être conçues comme le résultat des phénomènes sociolinguistiques, qui résultent de l'émergence des nouveaux modes de la communication numérique écrite à travers le contact via des écrans interposés.

Cette activité a conduit à de nouveaux comportements langagiers, marqués par des formes linguistiques et langagières, permettant ainsi l'intercompréhension entre des sujets n'ayant pas toujours les mêmes compétences linguistiques. De ce fait, les échanges dans les formules de salutations en ligne matérialisent une certaine organisation de la parole qui amène d'entrée en jeu les cyberscripteurs, à effectuer des choix linguistiques et langagiers. Nous remarquons également une dynamique notable de l'évolution des ressources langagières numériques dans la communication médiée par ordinateur, qui participe à une dynamique d'encodage et de décodage favorisant ainsi, le développement du répertoire « scripto-verbal plurilingue » des cyberscripteurs algériens. Il en ressort de cette étude que le réseau social Facebook privilégie l'usage des langues minoritaires (l'arabe algérien). À cet effet, le phénomène de l'alternance des langues s'explique par la relation amicale et conviviale entretenue entre les cyberscripteurs et/ou les langues (particulièrement l'arabe algérien) qu'ils mobilisent lors des échanges. Mais il permet également à ces derniers de remplir des objectifs identitaires en traçant des contours de la communauté sociolinguistique à laquelle ils appartiennent.

Enfin, l'alternance codique et (néo)codique, comme ressource langagière numérique, montre que le choix linguistique et/ou langagier dans les échanges synchrones sur Facebook est lié à des enjeux pratiques. Employée comme solution qui amène les cyberscripteurs algériens à des ajustements et à l'alignement des ressources langagières, l'alternance (néo)codique relève, de facto de formules entièrement ritualisées, de routines communicatives qui semblent être typiques à la communication médiée par ordinateur. Dès lors, peut-on considérer l'alternance (néo)codique comme une pratique spécifique à la communication médiée par ordinateur, et a priori un «we-code» des cyberscripteurs appartenant à une communauté sociolinguistique spécifique ? L'alternance (néo)codique est-elle l'expression des habitus comme signe d'appartenance à une communauté virtuelle spécifique à travers la communication en ligne?

\section{Bibliographie}

Ali-Bencherif M. Z. (2009). L'emploi alternatif de l'arabe algérien et du français dans des conversations bilingues: Modes de fonctionnement, régulation et ritualisation dans les séquences d'ouverture. Synergies Algérie, vol. 8, p. 79-89.

Ali-Bencherif M. Z. (2016). La cybercommunication : un espace d'échanges plurilingues, néo-pluri-graphiques et multiformes. Cahiers de linguistique, vol. 42, n 2, p. 31-38. 
Atifi H. (2007). Choix linguistiques et alternance codique dans les forums diasporiques marocains. In J. Gerbault (éd.), La langue du cyberespace: de la diversité aux normes. Paris : L'Harmattan, p. 31-46.

Auer P. (1995). The pragmatics of code-switching: A sequential approach. In L. Milroy \& P. Muysken (eds.), One Speaker Two Languages: Cross-Disciplinary Perspectives on Code-Switching, Cambridge: Cambridge University Press, p. 115-135.

Calvet L.-J. (2002). Mondialisation, langue et politiques linguistiques. Le versant linguistique de la mondialisation. Le français dans le monde, vol. 329, p. 39-42.

Chardenet P. (2005). Effets d'alternance et de mélanges dans les échanges plurilingues en ligne. In V. Pugibet et N. Gettliffe-Grant (coord.), Pluralité des langues et des supports: descriptions et approches didactiques, (Cahiers du français contemporain, vol. 10), Lyon : ENS Éditions, p. 237-253.

Develotte C. et Gee R. (2005). Contacts de 1@ngues sur écran ou comment on donne sa langue à la souris. In C. Van Den Avenne (éd.), Mobilités et contacts de langues, Paris : L'Harmattan, p. 147-161.

Develotte C., Kern R. et Lamy M.-N. (dir.) (2011). Décrire la conversation en ligne : le face à face distanciel, Lyon : ENS Éditions.

Goffman E. (1973). La mise en scène de la vie quotidienne. Tome 2. Les relations en public, Paris : Éditions de Minuit.

Gumperz J. J. (1982). Discourse Strategies. Studies in Interactional Sociolinguistics, Cambridge: Cambridge University Press.

Herring S. (1999). Interactional coherence in CMC. Journal of Computer Mediated Communication, vol. 4, $\mathrm{n}^{\circ}$ 4, [https://doi.org/10.1111/j.1083-6101.1999.tb00106.x].

Hymes D. (1972). Models of the interaction of language and social life. In J. J. Gumperz \& D. Hymes (eds.), Directions in Sociolinguistics. The Ethnography of Communication, New York: Holt, Rinehart \& Winston, p. 35-71.

Kerbrat-Orecchioni C. (2007). La «cyberpolitesse» formes de l'adresse ouverture et clôture dans les courriers électroniques. Quaderns de Filologia. Estudis Lingüistics, vol. 12 , p. $35-56$.

Liénard F. (2014). Les communautés sociolinguistiques virtuelles. Le cas des pratiques scripturales numériques synchrones et asynchrones mahoraises, Studii de lingvistică , vol. 4, p. $145-163$.

Marcoccia M. (2011). «T'es où maintenant?»: Les espaces de la conversation visiophonique en ligne. In C. Develotte, R. Kern et M.-N. Lamy (dir.), Décrire la conversation en ligne : le face à face distanciel. Lyon : ENS Éditions, p. 95-115.

Marcoccia M. (2016). Analyser la communication numérique écrite, Paris : Armand Colin.

Mondada L. (2007). Le code-switching comme ressource pour l'organisation de la paroleen-interaction. Journal of Language Contact, vol. 1, p. 168-195 [www. jlc-journal.org].

Pekarek Doehler S. (2011). Hallo! Voulez vous luncher avec moi hüt? Le "code switching" dans la communication par SMS. Linguistik online, vol. 48, $\mathrm{n}^{\circ} 4$, p. 49-70. [https://bop.unibe.ch/linguistik-online/article/view/333/499]. 
Pierozak I. (2010). Les corpus électroniques en sciences du langage : un eldorado ? Le discours et la langue, Revue de linguistique française et d'analyse du discours, vol. 2.1, p. 15-31.

Pothier M. (2004). Approche de la notion de ressources. In C. Develotte et M. Pothier (coord.), La notion de ressources à l'heure du numérique, (Notions en questions. Rencontres en didactique des langues vol. 8), Lyon : ENS Éditions, p. 15-22. 